\title{
Risk assessment of washout by slope flow along long-distance pipeline based on quantitative index cloud reasoning-integrated weighting
}

\author{
Manyin Zhang ${ }^{1}$, Zhizhong Sun ${ }^{1, *}$, Rong Xie ${ }^{1}$, Maocuo $\mathrm{Ka}^{1}$ and Shengxin Wang ${ }^{1}$ \\ ${ }^{1}$ Institute of Geological Hazards Prevention, Gansu Academy of Sciences, Lanzhou, Gansu 730000, China
}

\begin{abstract}
The washout by slope flow along long-distance oil \& gas pipelines is a common geological hazard that occurs when pipelines pass through the alluvial-proluvial fan section of mountain piedmont. Accurate and effective evaluation of the risk of single washout by slope flow is an important basis for disaster prevention and control decisions. According to the characteristics of the lack of basic research data in the development area of washout by slope flow, the complexity of the risk assessment structure and the strong randomness and ambiguity of the multi-index system, on the basis of rapid acquisition of initial data of indicators through field survey, simple experiment and sampling analysis, a quantitative index cloud reasoning risk evaluation model for slope flow washout of pipeline was established by introducing single-condition and single-rule cloud reasoning with summation integration weighting algorithm, and carry out instance verification. The evaluation results of 11 samples showed medium and relatively high risks, and the overall distribution trend is relatively concentrated. Compared with the results obtained by the entropy weightextension method and the standard recommendation method, the proposed method is more in line with the small-scale disaster background analysis and the reality of the study area, and it's also more beneficial to ensure the safe operation of pipelines. In this method, the obtainment of the source data is reliable, objective, and the preprocessing is simplified, the index weighting and classification are more reasonable, and the evaluation process takes into consideration of both the randomness and ambiguity of the system, which improves the accuracy and effectiveness of the evaluation results. It also provides a new way of thinking to other related research.
\end{abstract}

\section{Introduction}

Because of their high efficiency, all-weather, low cost, safety, and reliability, long-distance pipelines have become the main transmission channels for oil and natural gas resources. As of the end of 2020, the total mileage of long-distance oil \& gas pipelines in service in China has reached 156,000 kilometers. In response to the urgent needs of high-quality social and economic development and continuous optimization of the energy structure in the new era, it is expected that the mileage is to exceed 240,000 kilometers in 2025. China's pipeline industry will usher in an unprecedented "spring" of development, and its role in the national economy and people's livelihood will become more and more important ${ }^{[1-3]}$. However, shallow-buried long-distance oil \& gas pipelines are inevitably exposed to washout of runoff flow, debris flow, landslides, and special rock and soil mass (Collapsible loess, Salty soil, Swelling soil, Silt soft clay, etc.), due to long laying lines and passing through different geological, geographic, and climatic unit areas. Pipeline geological hazards occur due to adverse geological effects and harms, and the risk of these disasters will also become more complex and uncertain due to extreme climate changes, intensified engineering activities, and regional level upgrades along pipelines ${ }^{[4-6]}$. According to pipeline operators' investigations and assessments of the geological hazards of in-service oil \& gas pipelines in the Hexi Corridor section of Gansu province, known as the "China Oil \& Gas Pipeline Corridor," in the past ten years, pipelines on the piedmont proluvial fan are usually the most vulnerable to the impact of washout and erosion hazards (referred to as "washout by slope flow") on slopes that have not yet formed a fixed gully (valley), such as drop pit, rills, shallow ditches, and cut ditches. This kind of disasters are common (over $80 \%$ ) along the pipelines, and the damage is serious: They often cause insufficient buried depth of pipelines and exposure of pipeline optical cables; In severe cases, accidents such as cable breakage and exposure, suspended, or bent pipelines may occur, directly threatening the safe operation of the pipeline; It also exacerbates regional soil erosion and ecological environmental damage. Therefore, in the face of a large number of washout hazards along the pipeline with varying degrees of damage, after a scientific and effective quantitative evaluation of its risk, it is an effective means of controlling washout by slope flow of long-distance pipelines by designing the priorities of mitigation

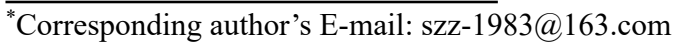


measures based on the risk status classification. It is also the key to preventing blind or inadequate investment and scientific management, which is of great significance to ensure the green and safe operation of pipeline projects ${ }^{[4-}$ 10].

China's pipeline transportation industry had a late start, but it has developed rapidly. There are few studies on pipeline geological hazard risks. At present, the focus is on the exploration of sub-links included in the oil \& gas pipeline integrity management system ${ }^{[4,11]}$. For pipeline geological disaster risk assessment, the most common practices are qualitative analysis and semi-quantitative artificial index assignment ${ }^{[5-7,9,12-15]}$ : On the one hand, it is subject to desert, Gobi, and remote factor of no man's land where the pipeline crosses, lacks of basic research data, the factors or indicators involved in the analysis are mostly from field observations, expert comments and scores, empirical data, background averages, etc., with few initial data and lack of objectivity and accuracy; On the other hand, in the evaluation model composed of "multi-level and multi-indicator", the calculation procedures are complex, the classification of index level is too "hard" etc., the ambiguity and randomness of the system are often ignored; Furthermore, the index weight is either based on cognitive interpretation or numerical reasoning. It fails to take into account the respective advantages of the two weighting methods. This paper intends to introduce the single-condition single-rule cloud reasoning and integration weighting by summation method, and acquisition of relevant measured data of the individual hazard of slope flow washout through fast and simple data mining methods, such as site survey, experiment, sampling analysis ${ }^{[9,14,16-19]}$. We proposed a quantitative index cloud reasoning evaluation model for the risk of slope flow washout for oil \& gas pipelines, to simplify the preprocessing procedure of quantitative data, soften the index boundary hard division, and also take into account the randomness and ambiguity of the evaluation system. The evaluation results are compared with the results obtained by the entropy weight-extension method and the standard recommendation method ${ }^{[6,14]}$ to verify the applicability and effectiveness of the built model, and in order to provide more scientific management and control of risks for slope flow washout of oil $\&$ gas pipelines. It also provides new research ideas and methods for relating researches.

\section{Main algorithms and evaluation steps}

\subsection{The single-condition single-rule cloud- reasoning algorithm}

Cloud theory ${ }^{[16-21]}$ is a new mathematical model that is used to solve the conversion between qualitative and quantitative concepts of things, and fully reflects the scientific processing of complex problems such as system ambiguity, randomness, and uncertainty by human thinking. It usually uses three digital characteristics of expected value $(E x)$, entropy $(E n)$, and hyper entropy $(H e)$ to control and calculate cloud droplets, which reflect the concept of language value of a certain thing, and it is realized by repeating calculations to generate a clustered cloud image that obeys Gaussian distribution conceptual cloud quantification. Conversely, a large number of cloud droplets within a certain range can be used to reversely derive cloud digital character values to achieve qualitative linguistics for quantitative problems. For an evaluation system composed of quantitative indicators, a singlecondition single-rule cloud reasoning process can usually be adopted, that is, the comprehensive use of $\mathrm{X}$ and $\mathrm{Y}$ condition cloud algorithms to achieve effective quantitative evaluation of multiple-indicator systems. The specific algorithm is as follows:

1) X-condition cloud: If the numerical features ( $E x, E n$, $H e$ ) of the concept and the quantitative value $x$ are known, a normal random number $E n^{\prime}$ with $E n$ as the expected value and $\mathrm{He}$ as the mean square error is generated; and the quantitative value $x$ is calculated as the certainty of the concept $y$ is:

$$
y=e^{\frac{-(x-E x)^{2}}{2\left(E n^{\prime}\right)^{2}}}
$$

2) Y-condition cloud: If the numerical features $(E x, E n$, $\mathrm{He}$ ) and certainty $y, y \in[0,1]$ of the concept are known, then a normal random number $E n^{\prime}$ with $E n$ as the expected value and $\mathrm{He}$ as the mean square error is generated; and calculate the quantitative value $x$ that satisfies the certainty $y$, namely:

$$
x=E x \pm E n^{\prime} \times \sqrt{-2 \ln y}
$$

The above algorithm integrates the cross advantages of fuzzy mathematics and probability statistics to ensure the effective transmission and inheritance of the fuzziness, randomness and uncertainty of things in the evaluation process. Compared with the general quantitative evaluation theory, it has obvious advantages and conforms to the complex nonlinear system's quantitative analysis and evaluation ${ }^{[20-22]}$

\subsection{Summation integration weighting method}

The cloud weighting $W_{c}{ }^{[20,23]}$ based on the golden ratio driving method can well reflect people's subjective cognition and interpretation ability, and also take into account the ambiguity and randomness of the subjects to be evaluated, but it ignores the interrelated and restrictive attributes between the indicators in the evaluation system, hence, there is no consideration of the integrity and unity of the evaluation system ${ }^{[24]}$. Similarly, the objective weight $W_{e}$ that we obtained based on mathematical operations such as entropy weight method can well reflect factors such as the amount of information of the assessment index, the degree of dispersion, and mutual relationship between the evaluation data, but it cannot reflect the cognitive interpretation ability of the decision maker, making the evaluation system too mechanized. Therefore, using the principle of summation integration ${ }^{[18]}$, the cloud weight $W_{c}$ and the entropy weight $W_{e}$ are additively integrated to obtain a new comprehensive weight $W$. This way, drawbacks in single-weight distribution can be avoided, and the subjective and objective integration and unification of index weighting can be realized, so that the distribution of weight 
coefficients is more scientific and reasonable. It is:

$$
W=a W_{c}+b W_{e}
$$

If there are $n$ indicators, then there are:

$$
\begin{gathered}
a=\frac{1}{n-1}\left(\sum_{i=1}^{n} i P_{i}-\frac{n+1}{n}\right) \\
b=1-a
\end{gathered}
$$

Where $a$ is the degree of influence of cloud weight $W_{c}$ on comprehensive weight $W ; b$ is the degree of influence of entropy weight $W_{e}$ on the comprehensive weight $W, P_{i}$ is the $i$-th component of cloud weight $W_{c}$ in ascending order.

\subsection{Risk assessment steps of a slope flow washout of pipeline by cloud reasoning- integrated weighting}

1) Construct individually a set of evaluation index factors that can comprehensively characterize a single slope flow washout risk of pipeline $U=\left\{u_{1}, u_{2}, \mathrm{~L} u_{n}\right\}$, a set of risk status comments $V=\left\{v_{1}, v_{2}, \mathrm{~L} v_{m}\right\}$, and a set of weights of index importance $W=\left\{w_{1}, w_{2}, \mathrm{~L} w_{n}\right\}$.

2) Divide and solve the cloud digital characteristic value of the evaluation index. The comment level scale of the index qui generally has an upper boundary value $x_{i j}^{1}$ and a lower boundary value $x_{i j}^{2}$. The expected value of each factor $i$ relative to the comment scale $j$ is:

$$
E x_{i j}=\left(x_{i j}^{1}+x_{i j}^{2}\right) / 2
$$

Since the upper and lower boundary values of the comment level scale $j$ are the boundary transition values of the adjacent two levels, they should belong to both of the two adjacent levels, and there is a certain fuzziness, so the scale boundary value relative to the adjacent membership scales should be equal, and the entropy of the cloud theory derived using $\exp \left[-\left(x_{i j}^{1}-x_{i j}^{2}\right) / 8\left(E n_{i j}\right)^{2}\right] \approx 0.5$ is:

$$
E n_{i j}=\frac{\left(x_{i j}^{1}-x_{i j}^{2}\right)}{2.335}
$$

Normally, the value of hyper entropy $H e_{i j}$ in cloud theory is based on experience ${ }^{[16]}$. The smaller the $H e_{i j}$, the smaller the degree of dispersion of the cloud droplets, and the thinner the normally distributed cloud layer formed; otherwise, the thicker it is.

3) Establish the subordination relationship matrix $R$ of each factor index $u_{i}$ to comment $v_{i}$. The quantitative parameters of each factor index and the cloud digital characteristics calculated in step 2) are implemented according to the single-condition and single-rule cloud generator of (1) and (2), using MATLAB programming to cycle 1000 times to determine the evaluation system index factor cloud reasoning transformation membership matrix $R=\left(R_{i j}\right)_{n \times m}$.

4) Complete the fuzzy relationship conversion operation between the weight set $W$ and the matrix $R$, and obtain the evaluation result set $B$ :

$$
B=W \otimes R=\left\{b_{1}, b_{2}, \mathrm{~L} b_{n}\right\}
$$

5) According to the principle of maximum degree of membership, the level corresponding to the $i$-th evaluation value with the maximum degree of membership is selected as the evaluation result; in order to make the level of the evaluation result more observable, the evaluation value with the maximum degree of membership can also be used in forward and reverse directions of the cloud generator ${ }^{[16]}$ to generate a cloud chart, which is then superimposed and compared with the ruler cloud of the total comment.

\section{Engineering case analysis}

\subsection{Evaluation source data acquisition}

The first line of the West-East Natural Gas Pipeline, a landmark project for the development of western China, runs eastward through the Gansu Hexi Corridor from Xinjiang, and most of the sections were laid along the cross-slope of the alluvial-proluvial fan (piedmont slope) on the northern slope of the Qilian Mountains. Over the past 20 years of operation, the pipeline projects have suffered from washout and erosion during the process of precipitation runoff discharge in the upstream area, and slope flow destruction has widespreadly developed. Especially in the $50 \mathrm{~km}$ section of the piedmont proluvial fan of Yumu Mountain in the south from Gaotai to Linze County, Zhangye City. The pipeline route is close to the foot of the mountain and crosses the fan surface. Various types of slope flow destruction, which are mainly as rills, shallow trenches, cut trenches and small gullies, etc., showed a trend of mass occurrence, with a development density of 1.1 locations $/ \mathrm{km}$, which has seriously affected the green and safe operation of pipelines. After a preliminary site investigation, 11 typical gullies (numbered WD1 $\sim$ WD11 respectively) were selected from 55 flood damages based on expert opinions. The initial data of the evaluation factors of washout by slope flow (Table 1 and Figure 1) were obtained by simple and fast methods such as detailed site surveys, pressurecontrolled Erosion experiments, Infiltration experiments,

\begin{tabular}{|c|c|c|c|c|c|c|c|c|c|c|c|}
\hline Evaluation index & WD1 & WD2 & WD3 & WD4 & WD5 & WD6 & WD7 & WD8 & WD9 & WD10 & WD11 \\
\hline Terrain slope $\left({ }^{\circ}\right)$ & 6.0 & 3.0 & 2.0 & 1.0 & 5.0 & 2.0 & 3.0 & 2.0 & 1.0 & 1.0 & 3.0 \\
\hline Vegetation cover $(\%)$ & 14.0 & 10.0 & 18.0 & 10.0 & 7.0 & 5.4 & 5.2 & 15.0 & 11.0 & 6.0 & 3.0 \\
\hline $\begin{array}{l}\text { Controlled particle size } d_{60} \\
(\mathrm{~mm})\end{array}$ & 9.09 & 9.09 & 10.0 & 7.69 & 11.11 & 12.50 & 5.00 & 14.29 & 4.76 & 2.00 & 7.14 \\
\hline Natural density $\left(\mathrm{g} / \mathrm{cm}^{3}\right)$ & 2.00 & 2.08 & 2.13 & 1.92 & 2.00 & 2.00 & 2.33 & 2.08 & 2.13 & 1.96 & 1.96 \\
\hline Permeability coefficient $(\mathrm{cm} / \mathrm{s})$ & 2.52 & 3.06 & 3.08 & 3.04 & 2.21 & 4.19 & 8.4 & 2.43 & 10.00 & 9.14 & 8.17 \\
\hline
\end{tabular}
soil sample particle size sieving and vegetation sample site statistics, then the risk assessment of slope flow washout along pipeline route based on cloud inferencing-integrated weighting was performed.

Table 1. Initial data of risk assessment index of washout by slope flow of pipeline 


\begin{tabular}{|c|c|c|c|c|c|c|c|c|c|c|c|}
\hline Anti-erosion index $\left(\times 10^{-3}\right)$ & 0.39 & 0.41 & 0.89 & 0.56 & 0.95 & 0.42 & 0.33 & 0.42 & 0.21 & 0.33 & 0.57 \\
\hline Maximum flow of gully $\left(\mathrm{m}^{3} / \mathrm{s}\right)$ & 17.5 & 4.33 & 9.24 & 79.8 & 66.43 & 119.35 & 15.78 & 20.8 & 26.44 & 52.31 & 29.9 \\
\hline $\begin{array}{l}\text { Distance between pipeline and } \\
\text { catchment entrance }(\mathrm{km})\end{array}$ & 1.45 & 1.22 & 0.86 & 3.45 & 3.45 & 3.70 & 0.32 & 0.27 & 0.23 & 0.37 & 0.24 \\
\hline $\begin{array}{l}\text { Angle between pipeline and } \\
\text { gully }\left(^{\circ}\right)\end{array}$ & 85.0 & 71.0 & 64.0 & 54.0 & 69.0 & 58.0 & 63.0 & 69.0 & 72.0 & 28.0 & 82.0 \\
\hline $\begin{array}{l}\text { Ratio of erosion depth and } \\
\text { pipeline burying depth }\end{array}$ & 0.17 & 0.11 & 0.05 & 0.14 & 0.10 & 0.11 & 0.52 & 0.44 & 0.21 & 0.4 & 0.33 \\
\hline $\begin{array}{l}\text { Hydraulic protection project } \\
\text { age(y) }\end{array}$ & 2.0 & 7.5 & 2.5 & 4.5 & 2.5 & 2.0 & 2.0 & 3.5 & 3.0 & 0.5 & 1.5 \\
\hline
\end{tabular}
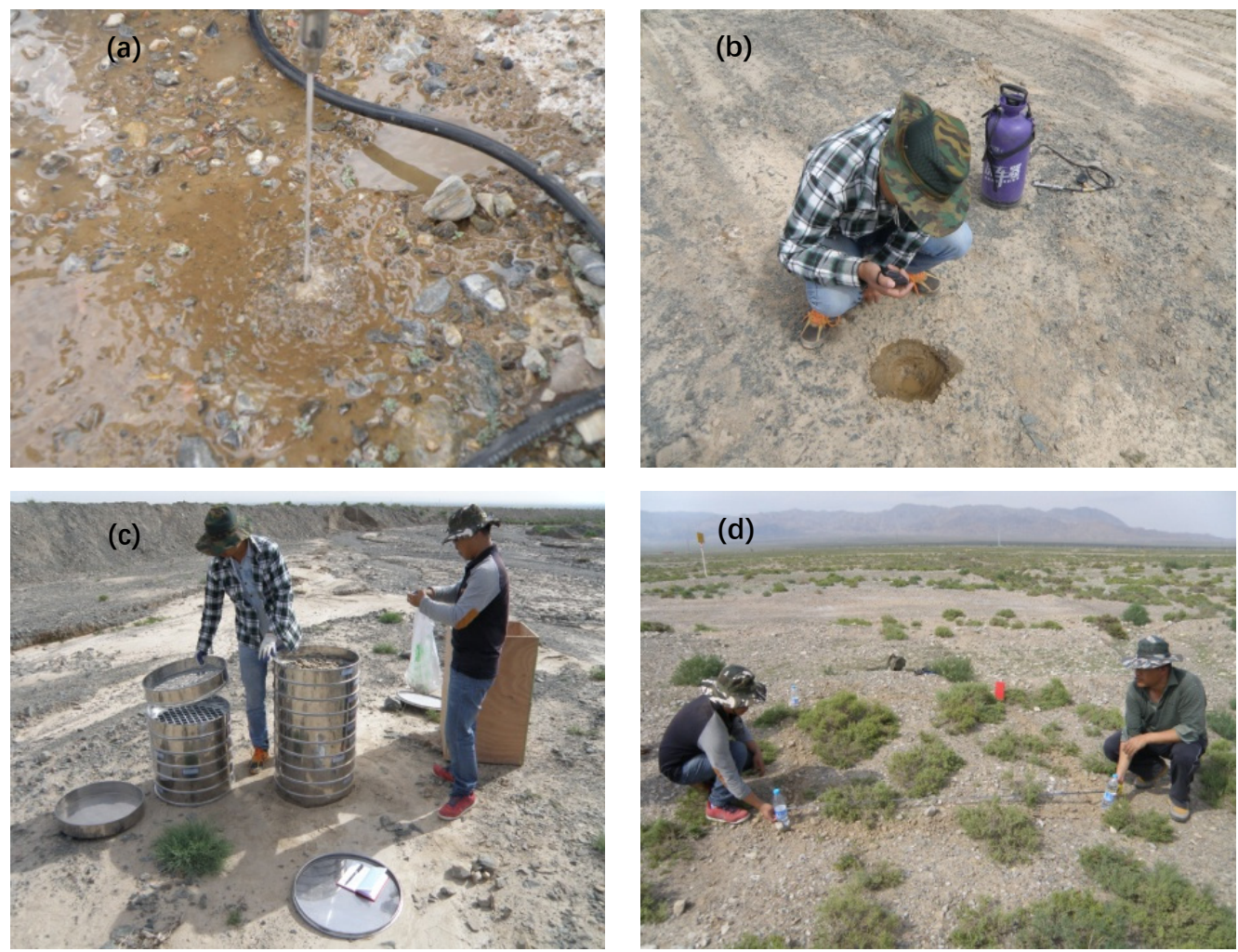

Figure 1. On-site acquisition of initial data for risk assessment index of slope flow washout of pipeline: (a) Erosion experiment; (b) Infiltration experiment; (c) Particle size sieving; (d) Statistics of vegetation sample site

\subsection{Establishment of evaluation organization system}

\subsubsection{Establish evaluation index system and its comment scales}

Combining relevant research results ${ }^{[5-12]}$, and comprehensive analysis of on-site investigations and simple tests, it is concluded that the risk level of slope flow washout of oil \& gas pipelines buried in shallow trenches on alluvial-proluvial fans is mainly controlled by the combined complex factors such as micro topography, rock and soil properties, precipitation runoff conditions, pipeline burying and protection. Starting from the principles of characterization of influencing factors, systematicness of evaluation, and the availability and accuracy of related data, 11 quantitative factors that are representative and consistent with aforementioned principles are selected as evaluation indicators from the above factors. The influencing factors and its representative index are shown in Table 2. According to the 5-level scale division principle recommended in the National Oil \& Gas Industry Standards ${ }^{[14]}$ and the scale division standard in the reference [6], the boundaries of selected comment scales of 11 indicators are divided as shown in Table 2.

Table 2. Risk assessment index system of washout by slope flow of pipeline

\begin{tabular}{|c|c|c|c|c|c|c|c|}
\hline \multirow[b]{2}{*}{$\begin{array}{l}\text { Evaluat } \\
\text { ion goal }\end{array}$} & \multirow[b]{2}{*}{$\begin{array}{l}\text { Influencing } \\
\text { factors }\end{array}$} & \multirow[b]{2}{*}{$\begin{array}{l}\text { Evaluation } \\
\text { index }\left(u_{i}\right)\end{array}$} & \multicolumn{5}{|c|}{ Index comments $\left(v_{i}\right)$ scale division } \\
\hline & & & $\begin{array}{l}\text { Low } \\
(E x, E n, H e)\end{array}$ & $\begin{array}{l}\text { Relatively Low } \\
(E x, E n, H e)\end{array}$ & $\begin{array}{l}\text { Medium } \\
(E x, E n, H e)\end{array}$ & $\begin{array}{l}\text { Relatively High } \\
(E x, E n, H e)\end{array}$ & $\begin{array}{l}\text { High } \\
(E x, E n, H e)\end{array}$ \\
\hline \multirow{3}{*}{ 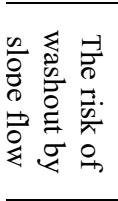 } & \multirow{2}{*}{$\begin{array}{l}\text { Micro } \\
\text { topography } \\
\text { conditions }\end{array}$} & Terrain slope $\left({ }^{\circ}\right)$ & $\begin{array}{l}0 \sim 1.0 \\
(0.5,0.43,0.01)\end{array}$ & $\begin{array}{l}1.0 \sim 2.0 \\
(1.5,0.43,0.01)\end{array}$ & $\begin{array}{l}2.0 \sim 3.0 \\
(2.5,0.43,0.01)\end{array}$ & $\begin{array}{l}3.0 \sim 4.0 \\
(3.5,0.43,0.01)\end{array}$ & $\begin{array}{l}4.0 \sim 10.0 \\
(7.0,2.57,0.01)\end{array}$ \\
\hline & & $\begin{array}{l}\text { Vegetation } \\
\text { coverage }(\%)\end{array}$ & $\begin{array}{l}0.5 \sim 1.0 \\
(0.75,0.21,0.01)\end{array}$ & $\begin{array}{l}0.3 \sim 0.5 \\
(0.40,0.09,0.01)\end{array}$ & $\begin{array}{l}0.3 \sim 0.1 \\
(0.20,0.09,0.01)\end{array}$ & $\begin{array}{l}0.1 \sim 0.05 \\
(0.05,0.0,0.01)\end{array}$ & $\begin{array}{l}0.05 \sim 0.001 \\
(0.03,0.02,0.01)\end{array}$ \\
\hline & $\begin{array}{l}\text { rock and soil } \\
\text { properties }\end{array}$ & $\begin{array}{l}\text { Control particle } \\
\text { Size } d_{60}(\mathrm{~mm})\end{array}$ & $\begin{array}{l}10.0 \sim 30.0 \\
(20.0,8.49,0.01)\end{array}$ & $\begin{array}{l}8.0 \sim 10.0 \\
(9.0,0.85,0.01)\end{array}$ & $\begin{array}{l}5.0 \sim 8.0 \\
(6.5,1.27,0.01)\end{array}$ & $\begin{array}{l}2.0 \sim 5.0 \\
(3.5,1.27,0.01)\end{array}$ & $\begin{array}{l}0.01 \sim 2.0 \\
(1.0,0.86,0.01)\end{array}$ \\
\hline
\end{tabular}




\begin{tabular}{|c|c|c|c|c|c|c|}
\hline & $\begin{array}{l}\text { Natural density } \\
\left(\mathrm{g} / \mathrm{cm}^{3}\right)\end{array}$ & $\begin{array}{l}3.0 \sim 4.0 \\
(3.5,0.43,0.01)\end{array}$ & $\begin{array}{l}2.5 \sim 3.0 \\
(2.8,0.21,0.01)\end{array}$ & $\begin{array}{l}1.5 \sim 2.5 \\
(2.0,0.43,0.01)\end{array}$ & $\begin{array}{l}1.0 \sim 1.5 \\
(1.3,0.21,0.01)\end{array}$ & $\begin{array}{l}0.1 \sim 1.0 \\
(0.55,0.38,0.01)\end{array}$ \\
\hline & $\begin{array}{l}\text { Permeability } \\
\text { coefficient }(\mathrm{cm} / \\
\mathrm{s})\end{array}$ & $\begin{array}{l}0.1 \sim 1.0 \\
(0.55,0.38,0.01)\end{array}$ & $\begin{array}{l}1.0 \sim 4.0 \\
(2.50,1.27,0.01)\end{array}$ & $\begin{array}{l}4.0 \sim 7.0 \\
(5.50,1.27,0.01)\end{array}$ & $\begin{array}{l}7.0 \sim 10.0 \\
(8.50,1.27,0.01)\end{array}$ & $\begin{array}{l}10.0 \sim 100.0 \\
(55.0,38.2,0.01)\end{array}$ \\
\hline & $\begin{array}{l}\text { Anti-erosion } \\
\text { index }\left(\times 10^{-3}\right)\end{array}$ & $\begin{array}{l}1.0 \sim 10.0 \\
(5.5,3.82,0.01)\end{array}$ & $\begin{array}{l}0.80 \sim 1.0 \\
(0.9,0.09,0.01)\end{array}$ & $\begin{array}{l}0.50 \sim 0.80 \\
(0.65,0.13,0.01)\end{array}$ & $\begin{array}{l}0.20 \sim 0.50 \\
(0.35,0.13,0.01)\end{array}$ & $\begin{array}{l}0.01 \sim 0.20 \\
(0.11,0.08,0.01)\end{array}$ \\
\hline \multirow{3}{*}{$\begin{array}{l}\text { Precipitation } \\
\text { Runoff } \\
\text { conditions }\end{array}$} & $\begin{array}{l}\text { Maximum } \\
\text { flow }\left(\mathrm{m}^{3} / \mathrm{s}\right)\end{array}$ & $\begin{array}{l}0 \sim 10.0 \\
(5.0,4.25,0.01)\end{array}$ & $\begin{array}{l}10.0 \sim 20.0 \\
(15.0,4.23,0.01)\end{array}$ & $\begin{array}{l}20.0 \sim 60.0 \\
(40.0,16.9,0.01)\end{array}$ & $\begin{array}{l}60.0 \sim 100.0 \\
(80.0,16.9,0.01)\end{array}$ & $\begin{array}{l}100.0 \sim 400.0 \\
(250.0,127.4,0.01)\end{array}$ \\
\hline & $\begin{array}{l}\text { Distance } \\
\text { between } \\
\text { pipeline and } \\
\text { catchment } \\
\text { entrance }(\mathrm{km})\end{array}$ & $\begin{array}{l}4.0 \sim 5.0 \\
(4.5,0.43,0.01)\end{array}$ & $\begin{array}{l}3.0 \sim 4.0 \\
(3.5,0.43,0.01)\end{array}$ & $\begin{array}{l}1.2 \sim 3.0 \\
(2.1,0.76,0.01)\end{array}$ & $\begin{array}{l}0.6 \sim 1.2 \\
(0.9,0.26,0.01)\end{array}$ & $\begin{array}{l}0.001 \sim 0.6 \\
(0.30,0.26,0.01)\end{array}$ \\
\hline & $\begin{array}{l}\text { Angle between } \\
\text { pipeline and } \\
\text { gully }\left({ }^{\circ}\right)\end{array}$ & $\begin{array}{l}0 \sim 20 \\
(10.0,8.49,0.01)\end{array}$ & $\begin{array}{l}20 \sim 40 \\
(30.0,8.49,0.01)\end{array}$ & $\begin{array}{l}40 \sim 60 \\
(50.0,8.49,0.01)\end{array}$ & $\begin{array}{l}60 \sim 80 \\
(70.0,8.49,0.01)\end{array}$ & $\begin{array}{l}80 \sim 90 \\
(85.0,4.25,0.01)\end{array}$ \\
\hline \multirow{2}{*}{$\begin{array}{l}\text { Pipeline } \\
\text { burying and } \\
\text { protection }\end{array}$} & $\begin{array}{l}\text { Ratio of erosion } \\
\text { depth and } \\
\text { pipeline } \\
\text { burying depth }\end{array}$ & $\begin{array}{l}0 \sim 0.1 \\
(0.05,0.04,0.01)\end{array}$ & $\begin{array}{l}0.1 \sim 0.2 \\
(0.15,0.04,0.01)\end{array}$ & $\begin{array}{l}0.2 \sim 0.4 \\
(0.30,0.08,0.01)\end{array}$ & $\begin{array}{l}0.4 \sim 0.6 \\
(0.5,0.08,0.01)\end{array}$ & $\begin{array}{l}0.6 \sim 1.0 \\
(0.8,0.17,0.01)\end{array}$ \\
\hline & $\begin{array}{l}\text { Hydraulic } \\
\text { protection } \\
\text { project age }(y)\end{array}$ & $\begin{array}{l}10 \sim 20 \\
(15.0,4.27,0.01)\end{array}$ & $\begin{array}{l}5.0 \sim 10.0 \\
(7.5,2.1,0.01)\end{array}$ & $\begin{array}{l}3.0 \sim 5.0 \\
(4.0,0.85,0.01)\end{array}$ & $\begin{array}{l}1.0 \sim 3.0 \\
(2.0,0.85,0.01)\end{array}$ & $\begin{array}{l}0 \sim 1.0 \\
(0.5,0.43,0.01)\end{array}$ \\
\hline
\end{tabular}

\subsubsection{Summation and integration weighting of indicator}

To ensure the scientific rationality of the indicators weight distribution, this paper adopts the weighting method of addition and integration of subjective and objective weight. Firstly, referring to relevant research ${ }^{[9,20,23]}$ and combining with the opinions of experts on pipeline geological hazards prevention, according to the importance of the 11 participating indicators on the risk of slope flow washout of pipeline, based on the 5-level weighting of extremely important, relatively important, general, less important, and unimportant to obtain subjective weight values (Table 3 ). Secondly, referring to the process of entropy weighting method and results in the reference [6], the 11 participating indicators are weighted by entropy weighting method to obtain objective weight values (Table 3 ). Finally, using formulas (3) (5) to perform the summation operation of subjective weighting (cloud weighting) and objective weighting (entropy weighting), and obtain in turn the integrated weight values of 11 indicators (Table $3)$.

Table 3. Weighting of 11 evaluation indices for slope flow washout of pipeline

\begin{tabular}{llll}
\hline Evaluation Index $\left(u_{i}\right)$ & $\begin{array}{l}\text { Subjective Weight } \\
\left(w_{c}\right)\end{array}$ & $\begin{array}{l}\text { Objective Weight } \\
\left(w_{e}\right)\end{array}$ & $\begin{array}{l}\text { Integrated } \\
\text { Weight }\left(w_{i}\right)\end{array}$ \\
\hline Terrain slope & 1.0 & 0.087 & 0.124 \\
Vegetation coverage & 0.309 & 0.092 & 0.062 \\
Control particle size $d_{60}$ & 0.50 & 0.107 & 0.086 \\
Natural density & 0.309 & 0.001 & 0.029 \\
Permeability coefficient & 0.50 & 0.086 & 0.078 \\
Anti-erosion index $\left(\times 10^{-3}\right)$ & 0.691 & 0.044 & 0.080 \\
Maximum flow & 1.0 & 0.177 & 0.158 \\
Distance between pipeline and catchment entrance & 0.691 & 0.189 & 0.135 \\
Angle between pipeline and gully & 0.309 & 0.015 & 0.034 \\
Ratio of erosion depth and pipeline burying depth & 1.0 & 0.112 & 0.134 \\
Hydraulic protection project age & 0.50 & 0.090 & 0.080 \\
\hline
\end{tabular}

\subsection{Result calculation and analysis}

\subsubsection{Result calculation}

First, according to the indicator comment scale in Table 2, the cloud digital characteristic values of the comment scale are calculated using formula (6) and (7) (Table 2). Then through the evaluation steps of 3 ) $\sim 5$ ), combining with the Integrated weight set in Table 3, using MATLAB software to perform single-condition single-rule cloud reasoning and related calculations, we obtain the result clouds of slope flow washout risk states of pipeline in 11 sites, and the digital characteristic values are shown in Table 4. Finally, through forward and reverse cloud generators of cloud theory ${ }^{[16]}$, the result cloud in Table 4 and the 5-level ruler cloud based on the golden section method are superimposed to generate the result cloud chart of risk levels of washout by slope flow of pipeline as shown in Figure 2. It can be seen obviously from Table 4 and Figure 5 that the risk statuses of slope flow washout in study area to the West-East Natural Gas Pipeline is WD11 $>$ WD10 $>$ WD7 $>$ WD9 $>$ WD1 $>$ WD5 $>$ WD6 $>$ WD2 $>$ WD4 $>$ WD3 $>$ WD8. The method in this paper can simultaneously solve the problem of visualization and sorting difficulty of risk status among parallel samples. 
Table 4. Risk assessment results of washout by slope flow of pipeline in 11 sites

\begin{tabular}{cccccccc}
\hline No. & $(E x, E n, H e)$ & No. & $(E x, E n, H e)$ & No. & $(E x, E n, H e)$ & No. & $(E x, E n, H e)$ \\
\hline WD1 & $(0.533,0.0121,0.0012)$ & WD2 & $(0.502,0.0191,0.0025)$ & WD3 & $(0.481,0.0206,0.0050)$ & WD4 & $(0.483,0.0258,0.0018)$ \\
WD5 & $(0.520,0.0161,0.0023)$ & WD6 & $(0.509,0.0121,0.0012)$ & WD7 & $(0.687,0.0142,0.0014)$ & WD8 & $(0.442,0.0192,0.0046)$ \\
WD9 & $(0.576,0.0252,0.0060)$ & WD10 & $(0.761,0.0138,0.0019)$ & WD11 & $(0.784,0.0204,0.0026)$ & & \\
\hline
\end{tabular}

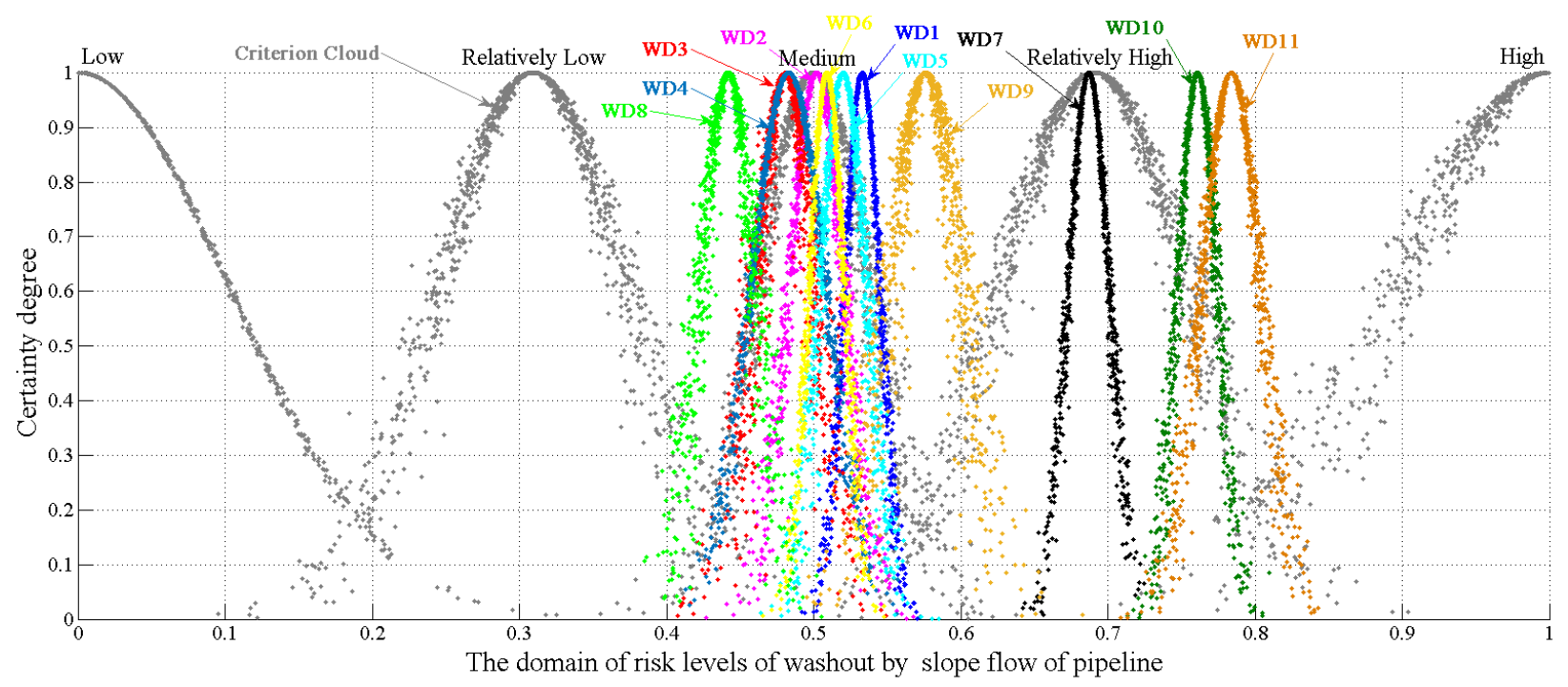

Figure 2. Cloud chart of risk levels of washout by slope flow of pipeline

\subsubsection{Comparison analysis}

In order to verify the applicability and effectiveness of the quantitative index cloud reasoning - integrated weighting method in the risk assessment of washout by slope flow of oil \& gas pipeline, the assessment results in this paper are compared and analyzed respectively with the results of the entropy weight-extension method and the standard recommendation method in the reference [6] (Table 5). The results show that: among the results obtained by this paper method, the risk levels of WD2 $\sim$ WD6 and WD8 are higher than the that of previous results, while the results of WD10 and WD11 are lower than that of the previous results. The occurrence of these inconsistent results is not only related to the optimization effect of the method in this paper on assessment objectives and weight determination, and the softening in the division of index scales, but also may be more affected by several highweight indicators such as terrain slope, runoff flow, distance from pipeline to catchment entrance, ratio of erosion depth and pipeline burying depth, age of hydraulic protection project. For example, the initial data of the three positive efficiency indicators ${ }^{[25]}$ such as slope, flow and distance in WD2 WD6 and WD8 are generally higher, and the initial data of the two negative efficiency indicators ${ }^{[25]}$ such as ratio of erosion depth and burying depth and age of hydraulic protection project overall are small or too long; whereas, the initial data of the highweight indicators for WD10 and WD11 are just the opposite of the above situation.

Table 5. Comparison of risk levels of washout by slope flow of pipeline with different evaluation methods

\begin{tabular}{llll}
\hline No. & $\begin{array}{l}\text { Results of Standard } \\
\text { Method }\end{array}$ & $\begin{array}{l}\text { Results of Entropy } \\
\text { Extension Method }\end{array}$ & \multicolumn{1}{c}{ Weight- } \\
\hline WD1 & Medium & Medium & Medium $\sim$ relatively high, incline to medium \\
WD2 & Relatively low & Relatively low & Medium $\sim$ relatively high, close to medium \\
WD3 & Relatively low & Relatively low & Relatively low $\sim$ Medium, near medium \\
WD4 & Relatively low & Relatively low & Relatively low $\sim$ Medium, near medium \\
WD5 & Medium & Relatively low & Medium $\sim$ relatively high, near medium \\
WD6 & Relatively low & Relatively low & Medium $\sim$ relatively high, close to medium \\
WD7 & Relatively high & Relatively high & Medium $\sim$ relatively high, close to relatively high \\
WD8 & Relatively low & Low & Relatively low $\sim$ Medium, incline to medium \\
WD9 & Medium & Medium & Medium $\sim$ relatively high, incline to medium \\
WD10 & High & High & Relatively high $\sim$ high, incline to medium \\
WD11 & Relatively High & High & Relatively high $\sim$ high, incline to medium \\
\hline
\end{tabular}

From Table 5, among the 11 sample results using different assessment methods, this paper results tend to be medium and relatively high, and the overall distribution of risk status trend of the samples is more concentrated. These results are mainly due to the fact that the conditions of atmospheric precipitation and underlying surface are 
basically the same in the small-scale disaster-pregnant environment of the study area (east-west $50 \mathrm{~km}$, northsouth $10 \mathrm{~km}$ ), such as small differences in topography conditions, rock and soil properties, precipitation runoff conditions, pipeline route and other influencing factors. While the results of the entropy weight-extension method and the standard recommendation method are scattered, ranging from low or relatively low to high 5-level status distribution, and most of them are below the relatively low level. This information provides decision makers with the basis for judging the overall relatively low risk of washout by slope flow of pipeline, which is not consistent with the above actual situation, and is not conducive to the safe operation of pipelines. In addition, it can be seen from Figure 5 and Table 5 that superimposing the assessment result cloud chart and the comment cloud chart is conducive to the fine analysis of the risk status of the sample to be evaluated. The results of the standard method and the entropy weight-extension method in Table 5 are compared with the results of this paper (Comparison of the same row in the table); Or among samples results of this paper (Comparison of the fourth column in the table) are compared. The assessment results at the same level, the cloud results can be described by "incline, near, close to " or the cloud drops distribution can be more refined in describing the distribution of assessment levels. It improves the accuracy of the assessment results to a certain extent, and provides more guidance for further analysis and decision-making.

\section{Conclusion}

1) The factors affecting the risk of washout by slope flow along oil \& gas pipeline route are complex and diverse. The initial data of evaluation factors are few, and the evaluation system has strong randomness and ambiguity. Common evaluation processes rely on expert scores, background averages, regional data and so on in a source data acquisition, and the evaluation model cannot deal with the ambiguity and randomness of the system at the same time. In this paper, on the basis of quick and easy data mining, such as site survey, experiment and sampling analysis, a risk assessment model of washout by slope flow of a pipeline based on quantitative index cloud reasoning-integrated weighting is constructed. Through empirical analysis of 11 slope flow washout in the alluvialproluvial fan section of Yumu Mountain in the Hexi Pipeline Corridor, the applicability and effectiveness of the established evaluation model have been verified.

2) The assessment index data comes from field experiments, surveys and measurements, and the initial data sources are objective and reliable; by adding and integrating cloud weight and entropy weight, the index comprehensive weight can avoid the drawback of subjective randomness and objective machinery of single weight to a certain extent.

3) The single-condition single-rule cloud generator algorithm for cloud conversion of quantified data weakens the hard division of hierarchical boundaries, simplifies the preprocessing of initial data, ensures the effective transmission and inheritance of system fuzziness, randomness and uncertainty, and realizes the scientific quantitative analysis of complex nonlinear systems.

4) The assessment results are cloud or cloud drops chart composed of three parameters: expected value, entropy, and hyper entropy. Through the process of superposition and comparison etc., functions such as sorting, screening, accurate analysis, and visual expression of the samples to be evaluated can be realized. It provides high-quality scientific and technological support for monitoring and prevention, planning and decision-making of washout disaster by slope flow of oil $\&$ gas pipeline, and also proposes a set of scientific and effective new methods for related research.

\section{Author in Brief}

ZHANG Manyin, Associate Researcher, Master Degree, Main Engaged in Prevention Research of Geological Hazards. Email: 42062509@qq.com

\section{Acknowledgments}

Supported by the Second Tibetan Plateau Scientific Expedition and Research Program (STEP, Grant No. 2019QZKK0902); The Applied Research and Development Project of Gansu Academy of Sciences (Grant No. 2018JK-14, 2019JK-05); The Youth Science and Technology Fund of Gansu Province (Grant No. 18JR3RA252); The National Natural Science Foundation of China (Grant No. 42067066); The Innovation Team Project of Gansu Academy of Sciences (Grant No. CX201801).

\section{References}

1. Zou C N, Zhao Q, Chen J J, et al. Natural gas in China: Development trend and strategic forecast[J]. Natural Gas Industry, 38(4): 1-11 (2018).

2. Zhu Q Z, Li Q Y, Wu C, et al. Construction Status and Development Trend of Global Oil \& Gas Pipelines in 2017[J]. Oil \& Gas Storage and Transportation, 38(4): 373-378 (2019).

3. Wang H J, Zhu Q Z, Zhang Y P. Overview of Oil and Gas Pipelines in the World[J]. Oil \& Gas Storage and Transportation, 34(1): 15-18 (2015).

4. Yao A L, Zhou L G, Wang L, et al. Management and Risk Evaluation on Long-distance Gas Pipelines Related to Regional Level Upgrading[J]. Natural Gas Industry, 37(1): 124-130 (2017).

5. Zhang J Y, Du J S. Analysis on the Pipeline Rupture Induced by Flood[J]. Oil \& Gas Storage and Transportation, 19(1): 16-19 (2000).

6. Zhang Q, Wang S X, Wang P F, et al. Assessment of Risk of Flood Damage at Slope of Pipeline Based on Combination of Entropy Method and Extension Theory[J]. China Safety Science Journal, 27(12): 110115 (2017).

7. Xiong J N, Sun M Y, Sun M. Risk Assessment on Mountain Torrents and Debris Flows along Lon- 
distance Pipelines Based on the GIS and CouplingCoordination Principle[J]. Natural Gas Industry, 37(1): 116-124 (2017).

8. Zhao B S, Wang S X, Xu K, et al. Experimental Study of Drop Pit Characteristics of Rill Erosion on Foreland Pluvial Fan Slope land[J]. Arid Land Geography, 40(2): 348-354 (2017).

9. Zheng Q C, Yao A L, Guan H P, et al. Safety Evaluation on Flood Damage at Slope of Oil and Gas Pipelines Based on Cloud Model[J]. Journal of Safety and Environment, 12(4): 234-238 (2012).

10. Wang R, Zhao F. Application of Slope Water Damage Prevention and Control Technology in Hengduan Mountain Area[J]. Chinese Journal of Underground Space and Engineering, 13(S2): 896-900 (2017).

11. Wang X, Duan Q Q. Improved AHP-TOPSIS Model for the Comprehensive Risk Evaluation of Oil and Gas Pipelines[J]. Petroleum Science, 16: 1479-1492 (2019).

12. Huang J C, Meng C Z. Analysis on Flood Damage from Crossing River Pipeline Project[J]. Journal of Sediment Research, (2): 41-49 (1998).

13. Jing H Y, Hao J B, Chen Y J, et al. Technique and Application of Geologic Hazard Risk SemiQuantitative Assessment of Pipeline[J]. Oil \& Gas Storage and Transportation, 30(7): 497-500 (2011).

14. A National Energy Administration, People's Republic of China. (SY/T 6828-2017) Technical Specification for Geological Hazards Risk Management of Oil and Gas Pipeline [S]. Petroleum Industry Press Ltd, Beijing (2018).

15. Du M, Zhao D F, Meng Y F. A Multi-level Extension Evaluation on Failure Risk of Long-Distance Gas Pipeline in Landslide Area[J]. Oil \& Gas Storage and Transportation, 31(8): 564-567 (2012).

16. Li D Y, Du Y. Artificial Intelligence with Uncertainty $\left(2^{\text {nd }}\right.$. End $)[\mathrm{M}]$, National Defense Industry Press, Beijing (2014).

17. Zhang L M, Wu X G, Chen Q Q, et al. Developing a Cloud Model Based Risk Assessment Methodology for Tunnel-Induced Damage to Existing Pipelines[J]. Stoic Environ Res Risk Assess, 29(2): 513-526 (2015).

18. Qu Z Y, Wang J, Wu Y, et al. Synthetic Evaluation Method for Electric Power Production Safety Based on the Extension Cloud Model[J]. Safety and Environmental Engineering, 22(3): 94-98 (2015).

19. Guo Y B, Meng X L, Meng T, et al. A Novel Method of Risk Assessment Based on Cloud Inference for Natural Gas Pipelines[J]. Journal of Natural Gas Science and Engineering, 30: 421-429 (2016).

20. Zhang $M$ Y, Wang $S \mathrm{X}$, Sun $Z$ Z, et al. Comprehensive Evaluation of Landslide Risk of Oil and Gas Pipelines Based on Cloud Theory[J]. Chinese Journal of Engineering, 40(4): 427-437 (2018).

21. Zhao D F, Li C B, Wang Q Q, et al. Comprehensive Evaluation of National Electric Power Development Based on Cloud Model and Entropy Method and
TOPSIS: A Case Study in Countries[J]. Journal of Cleaner Production, 277: 123190-123203 (2020).

22. Fu J L, Chen W B, Shao Y W, et al. Evaluation on Flood Damage Degree of Farmland Based on Normal Cloud Theory[J]. China Land Science, 33(2): 76-84 (2019).

23. Fu H L, Huang Z, Huang H W, et al. Health Diagnosis Method of Shield Tunnel Structure Based on Cloud Theory[J]. Chinese Journal of Engineering, 39(5): 794-801 (2017).

24. Chen S Q, Chen H Y, Wu X G, et al. Risk Assessment of Metro Tunnel Leakage Based on Copula-cloud Model[J]. Journal of Civil Engineering and Management, 36(5): 90-95 (2019).

25. Li T, Li F W, Li X X, et al. Comprehensive Assessment of Earthquake Hazard Risk Based on Normal Cloud Model - A Case Study of Bijie City[J]. China Safety Science Journal, 25(10): 166-171 (2015). 